\title{
CALCULATION OF NET EMISSION COEFFICIENT IN Ar-Cu AND SF 6 -Cu THERMAL PLASMAS
}

\author{
A. GLEIZES, J.J. GONZALEZ, B. LIANI and B. RAHMANI \\ Laboratoire Décharges dans Ies Gaz, URA n'277, CPAT, Université Paul \\ Sabatier, 118 route de Narbonne, F-31062 Toulouse Cedex, France
}

Résumé - Nous avons calculé le coefficient d'émission nette dans des plasmas thermiques établis dans des mélanges $\mathrm{SF}_{6}-\mathrm{Cu}$ et $\mathrm{Ar}-\mathrm{Cu}$, pour $\mathrm{T}>5000 \mathrm{~K}$. Le transfert radiatif des raies à été calculé par l'intermédiaire du facteur de fuite et nécessite l'étude des profils des raies. Les résultats montrent l'importance décisive de la vapeur métallique sur le rayonnement du plasma à basse température. L'absorption des raies du cuivre est du même ordre que celle des raies du soufre et de fluor dans les mélanges $\mathrm{SF}_{6}-\mathrm{Cu}$. Par contre dans les mélanges $\mathrm{Ar}$ - $\mathrm{Cu}$, elle est différente de l'absorption des raies d'argon.

Abstract - Net emission coefficient was calculated for thermal plasmas in $\mathrm{SF}_{6}$-Cu and Ar-Cu mixtures $(\mathrm{T}>5000 \mathrm{~K})$. Continuum and line emissions were taken into account. Radiative transfer of each line was computed by means of an escape factor depending on the shape and on the broadening of the line. Results showed the essential influence of metal vapor on plasma radiation for the lowest temperatures. The copper line absorption is analogous to that of sulfur and fluorine lines $\left(\mathrm{SF}_{6}\right.$ plasma) but different from that of argon lines.

\section{I - INTRODUCTION}

Overall modelling of arcs or thermal plasmas of the sort found in switchgear and in physical-chemical processes requires a good understanding of the thermodynamic properties and transport coefficients. The properties are generally calculated assuming local thermodynamic equilibrium (LTE). However even then, there is one property which remains difficult to calculate : the net emission coefficient, $\varepsilon_{N}$, which corresponds to the locally radiated power and which takes account of autoabsorption of the radiation. Strictly, we can not separate the calculation of $\varepsilon_{\mathrm{N}}$ from the calculation of the temperature profile. But, coupling the radiative transfer equation with those of conservation of mass, momentum and energy would lead to extremely tedious computation. Certain simplifications are therefore made to the radiation term. In the present study we report the calculation of the net emission coefficient, as defined by Lowke $/ 1 /$, in which it is assumed that the plasma is isothermal at temperature T.

In spite of the simplification, the values of $\varepsilon_{N}$ have been only calculated for a very reduced number of gases over the temperature and pressure ranges of thermal plasmas. This is due to the fact that the calculation is very strongly dependent on the species involved (energy levels, oscillator strengths) and line broadening and thus on the conditions of the plasma. We calculated the values of $\varepsilon_{N}$ for mixtures of gas and metal vapour often found in industrial applications since arcs are often seeded with metal vapours coming either from erosion of the electrodes and walls or from actual injection of metal powder into the arc.

The presence of metal vapours can considerably modify the properties of thermal plasmas. Their low ionisation potential tends to modify the electrical conductivity $\sigma$ and thus the temperature profile. $\sigma$ was calculated for numerous gases and mixtures. Concerming radiation, we know qualitatively and experimentally that the presence of metal vapours tends to increase energy losses but little quantitative data is available for gas-metal vapour mixtures $/ 2-3 /$. In the present work we chose two types of mixtures. One is found in strong current circuit-breaker arcs i.e. the mixture $\mathrm{SF}_{6}-\mathrm{Cu}$. Calculations were performed over the temperature range 6000 to $30000 \mathrm{~K}$ and for pressures between 1 and $8 \mathrm{~atm}$. The other mixture studied, $\mathrm{Ar}-\mathrm{Cu}$ occurs in laboratory transferred arcs with a copper electrode and in ICP torches. Calculations were done fo: atmospheric pressure over the temperature range 6000 to $25000 \mathrm{~K}$. 


\section{II - BASIS OF THE CALCULATIONS}

To account for autoabsorption of radiation by the plasma we calculaied the net emission coefficient $\varepsilon_{N}$ defined by Lowke / $/$ : it is the fraction of power radiated, per unit volume and solid angle, at the centre of an isothermal sphere of radius $R$, which escapes from the sphere :

$$
\varepsilon_{N}=\int_{0}^{\infty} B_{v} K_{v}^{\prime} \exp \left(-K_{v}^{\prime} R\right) d v
$$

$B_{v}$ is Planck's function $K_{v}$ the absorption coefficient corrected for the effect of stimulated emision. According to Lowke /1/ eq. 1 can be used to calculate the net emission coefficient relative to the axis of a cylinder of radius $R$. In our calculation we assume that the plasmas is isothermal and homogeneous. We also assume that plasma is in LTE and thus that Kirschhoff's law is valid. The main difficulty is to determine the variations of the absorption coefficient with the frequency $v$. Account must be taken of the mechanisms of continuum emission and of the line profiles.

\section{- Continuum}

Continuum phenomena are bremsstrahlung, radiative attachment and recombination. Radiation due to slowing down of the electrons is not preponderant in thermal plasmas. We used the semi-classical theory for the electronneutral interaction and the hydrogenoid approximation for the electron-ion interaction /4-5/.The coefficient of emission due to radiative attachment was calculated using the photodetachment cross-sections of the $\mathrm{S}$ - and $\mathrm{F}$ - ions given by Robinson and Geltman $/ 6 /$.

The largest contribution to the continuum is given by radiative recombination. For sulphur, fluorine and argon we used the values of the emission coefficient deduced by the method of the Scaled Thomas-Fermi Potential (STFP). For argon the values were given by Hofsaess $\pi /$ and for sulphur and fluorine we used those of Gongassian $/ 8 \%$. For copper, the hydrogenoïd approximation was developed.

\section{- Line emission and broadening}

To determine the spectral variations of the absorption coefficient of a line, the total absorption (or emission) coefficient and the profile of the line must be known. The total coefficient is given by the composition of the plasma and by the oscillator strength. Generally, the values of oscillator strength given by the NBS were chosen. Then, a large number of additional lines were taken into account. The oscillator forces for sulphur and fluorine by Gongassian /8/ were calculated using the STFP method and for copper they were listed by Kurucz and Peytremann $19 \%$.

To determine the line profiles, we studied various causes of broadening. The effects of pressure were dealt with using semi-classical theories /10/. So, Van der Waals broadening (perturbation by a neutral atom) was calculated using impact approximation with an $\mathrm{r}^{6}$ interaction potential . Resonance broadening involves the interaction between two similar atoms when the upper level of the emited line is coupled to the ground state via a dipolar transition. The half-width depends on the oscillator strength of the resonant level. Among the effects of pressure on thermal plasmas, that of Stark is the strongest : it appears when the disturbance arises from a charged particle. With an interaction potential bearing the term $r^{4}$ (quadratic Stark effect) the breadth of the profile is dependent on any transition involving emitter and absorber levels of the line in question.

The pressure effect line profiles can, well within the accuracy of the approximations used here, be assimilated to the Lorentz type. With Doppler widening a Voigt-type profile is reached.

\section{Escape factor}

For the purposes of calculation we made an additional and important hypothesis : we assumed that the lines are isolated i.e. that line overlapping has a negligible effect on the radiative transfer of the lines. In this way, each line can be dealt with separately enabling the introduction of the notion of escape factor, $\Lambda$, which is the ratio of the radiation escaping the plasma to that of a transparent plasma. The relevance of the escape factor is that it only depends on a limited number of parameters. It can be demonstrated that if the profile of the line is purely Lorentzian, $\Lambda$ is proportional to the optical depth at the centre of the line $\tau_{0}$. For a Voigt profile $\Lambda$ depends both on $\tau_{\mathrm{o}}$ and on the ratio of Lorentz to Gaussian broadening /11/: 


$$
\Lambda=2 \int_{0}^{\infty} P_{1}(v) \exp \left[-\tau_{o} \frac{P_{1}(n)}{P_{1}(0)}\right] d v
$$

where $P_{1}$ is the nomalized Voigt profile given by :

$$
P_{1}(v)=\frac{a}{\pi^{3 / 2} \Delta v_{D}} \int_{-\infty}^{+\infty} \frac{\exp -\left(\frac{y}{\Delta v_{D}}\right)^{2}}{a 2+(y-v)^{2}} d y
$$

with a the (half) half-width of Lorentz broadening and $\Delta v_{D}$ the characteristic width of the Gaussian profile. We performed a preliminary calculation of $\Lambda$ as a function of these parameters to obtain a table of numerical values easily useable in the general calculation.

\section{III - RESULTS FOR PURE ARGON AND SE}

\section{III-1 Pure argon}

Many results have been reported in the literature. From an experimental point of view, comparisons can only be made for the detectable portion of the radiation, i.e. when $\lambda>200 \mathrm{~nm}$. Table 1 lists the values we calculated above $\lambda=200 \mathrm{~nm}$ for three different temperatures and compares them with the experimental values of Evans and Tankin /12/ and Yakubov /13/. The main contribution for $\lambda<200 \mathrm{~nm}$ arises from resonance lines. The lines are both very intense and strongly absorbed. Figure 1 plots the total emission coefficient of argon at atmospheric pressure, without auto-absorption $(R=0)$, the involvement of resonance lines in the radiation, the net emission coefficient for $R=0.2 \mathrm{~cm}$ and lastly the contribution of the resonance lines at $R=0.2 \mathrm{~cm}$. Very strong absorption of the resonance lines is observed : the escape factor is lower than $10^{-3}$ when $\mathrm{T} \leq 10000 \mathrm{~K}$ and is equal to about 0.016 when $T$ is $15000 \mathrm{~K}$. The factor increases with temperature owing to the decrease in the density of the ground-state atoms and especially to the broadening of the lines through Stark effect. With the exception of these resonance lines, self-absorption of the remaining radiation is fairly low.

\begin{tabular}{|cccc|}
\hline$T$ & Yakubov $/ 12 /$ & Evans $/ 13 /$ & Our results \\
\hline 12000 & 87 & 57 & 63 \\
\hline 14000 & 284 & 275 & 209 \\
\hline 17000 & 383 & 385 & 258 \\
\hline
\end{tabular}

Table 1 : Comparison of emission coefficient $\left(W / \mathrm{cm}^{3} / \mathrm{st}\right)$ for $\lambda>200 \mathrm{~nm}$.

\section{III-2 Pure $\mathrm{SF}_{6}$}

The only values in the literature for the net emission coefficient are those calculated by Liebermann and Lowke /14/. Figure 2 compares our results with those of /14/ for SF6 at atmospheric pressure. Two types of comparison are made : between radiation values without auto-absorption $(R=0)$ and between net emission coefficients at $R=0.2 \mathrm{~cm}$. Itcan be noted that although our values for $R=0$ are lower than those in $/ 14 /$, the opposite is true when self-absorption is taken into account. Two explanations can be given. The first bears upon our hypothesis of no line overlapping : this leads to an overestimation of the net emission coefficient. Yet, we did perform a general calculation taking line overlapping into account and the values we found were at most $15 \%$ greater than the values calculated using the escape factor. Now, the difference between the two series (ours and those of $/ 14 /$ ) at $R=0.2 \mathrm{~cm}$ is greater than $15 \%$. The second explanation would be an underestimation of the results by Liebermann and Lowke.

The various contributions to the net emission coefficient in pure $\mathrm{SF}_{6}$ are represented in figure 3 for $\mathrm{R}=0.2 \mathrm{~cm}$. The escape factor of the resonance lines for sulphur and fluorine (both neutral and ionized species) is less than 1 $\%$ for $T \leq 8000 \mathrm{~K}$ but is of the order of 0.10 for $\mathrm{T}>10000 \mathrm{~K}$. So, the contribution of these lines is not negligible even taking account of auto-absorption in the plasma. Finally let us note that, for a given temperature, the net radiation of an $\mathrm{SF}_{6}$ plasma is much more intense than that of an argon plasma. 


\section{IV - INFLUENCE OF COPPER}

The influence of the proportion of copper on the radiation is illustrated in figures 4 and 5 which plot the variations of the net emission coefficient in $\mathrm{Ar}-\mathrm{Cu}$ and $\mathrm{SF}_{6}$-Cu mixtures for different concentrations (per volume) of copper. The first observation that can be made is that the influence of copper is strongest at the low temperatures. This is due to the low ionization potential of the metal. It can also be noted that the influence of copper is stronger in argon than in $\mathrm{SF}_{6}$. At low temperatures the radiation due to sulphur is much greater than that from argon because of differences of electronic configuration (mainly energies of excitation and ionization) so the presence of copper is felt much more in argon. At high temperatures the degree of ionization is relatively independent of the type of gas or the vapour present : $\varepsilon_{\mathrm{N}}$ is therefore not greatly affected by the species present, even copper.

It should also be noted that for the same nominal proportion of copper in a mixture, there is not actually the same quantity of copper in an argon mix as in a $\mathrm{SF}_{6}$ mix. The proportion is defined per volume (or per mole) of initial molecules. Thus in an argon-copper mixture the partial pressure of copper, $\mathrm{P}_{\mathrm{Cu}}$, is equal to :

$$
\mathrm{P}_{\mathrm{Cu}}=\mathrm{P}-\mathrm{P}_{\mathrm{c}}-\mathrm{P}_{\mathrm{Ar}}
$$

where $P_{e}$ is the partial pressure of the electrons and $P$ the total pressure.

However, for an $\mathrm{SF}_{6}$-copper mixture we have :

$$
P_{C_{u}}=P-P_{e}-P_{S}-P_{F}
$$

Considering the decomposition of the $\mathrm{SF}_{6}$ molecule at high temperature, the partial pressure of copper is much lower in the mixture with $\mathrm{SF}_{6}$ than in the argon mixture for the same initial proportions. This explains for example that at $\mathrm{T}=8000 \mathrm{~K}$ the radiation of a mixture $90 \% \mathrm{Ar}-10 \% \mathrm{Cu}$ is twice as high as that of a mixture $90 \% \mathrm{SF}_{6}-10$ $\%$ Cu.

One very interesting result concerns the relative absorption of the copper radiation with respect to that of the plasmagenic gas. This is illustrated in figure 6 for $\mathrm{SF}_{6}-\mathrm{Cu}$ where the relative contribution of the radiation due to copper is plotted. The solid line gives the result for non-absorbed radiation $(R=0)$ and the dotted line for $R=0.5$ $\mathrm{cm}$. For a given copper content, when the solid curve is below the dotted curve, the absorption of the radiation from copper is stronger than that of radiation from $\mathrm{SF}_{6}$. When the copper content is low $(<1 \%)$ the radiation from the copper is absorbed less owing to the low concentration of absorbing atoms. However; when the copper content reaches $10 \%$, its radiation is more strongly reabsorbed as long as $T \leq 16000 \mathrm{~K}$. At higher temperanures this trend is reversed owing to the strong absorption of the resonance lines of fluorine and of the ionic species $\mathrm{S}^{+}$, $\mathrm{F}^{+}$and $\mathrm{S}^{++}$. For the $\mathrm{Cu}$-Ar mixture, the results are presented in figure 7 ; the radiation of argon shows greater absorption owing to the properties of the resonance lines of argon.

For a given proportion of copper, the influence of the thickness of the plasma is illustrated in figures 8 and 9. Overall, there is a strong absorption at low temperatures corresponding to high densities of absorbing atoms and to lines which have not undergone much broadening (the wider a line is the less it is absorbed). It can also be seen that the values of $\varepsilon_{N}$ are hardly dependent on $R$ as soon as $R$ is greater than 1 or $2 \mathrm{~mm}$.

The influence of pressssure on the radiation of $\mathrm{SF}_{6}-\mathrm{Cu}$ is shown in figures 10 and 11 . The coefficient $\varepsilon_{\mathrm{N}}$ is more or less proportional to the pressure. Finally, in figure 12, the influence of copper on the radiation of argon is compared to that of iron. The values for iron were taken from $/ 3 /$.

\section{$\mathrm{V}$ - CONCLUSION}

Net emission coefficient was calculated for two mixtures in homogencous and isothermal plasmas. The accuracy of the results is limited by that of oscillator strengths, by the approximate theories used in continuum calculation and especially by the theories of line broadening. In particular the hypothesis relative to the line wing profile may lead to errors in the radiative transfer of strongly absorbed lines. The total accuracy on the net emission coefficient is estimated to a factor of two.

Nevertheless the influence of copper vapour is often higher than this inacccuracy for low temperature values ( $\mathrm{T}<8000 \mathrm{~K}$ for $\mathrm{SF}_{6}$ abd $\mathrm{T}<10000 \mathrm{~K}$ for $\mathrm{Ar}$ ). Then, taking into account the $\varepsilon_{\mathrm{N}}$ values given here may strongly modify the temperature fields computed by means of a total modelling, as it has been demonstratcd in a particular example by Proulx et al /15/.

\section{REFERENCES}

/1/ LOWKE, J.J., J. Quant. Spectrosc. Radiat. Transfer, 14 (1974) 111. 
/2/ CRAM, L.E., J. Phys. D 18 (1985) 401.

13/ ESSOLTANI, A, PROULX, P., GLEIZES, A. and BOULOS, M.I., 1990 Int. Conf. Winter Conf. (Jan. 1990) and paper submitted in J.A.A.S. (1990).

14. CABANNES, F. and CHAPELLE, J., "Spectroscopic plasma diagnostic" in "reactions under plasma conditions", vol 1, ch 7, Wiley Interscience, New-York (1971).

15/ GLEIZES, A., GONGASSIAN, M. and RAHMANI B., J. Phys. D 22 (1989) 83.

16/ ROBINSON, E.J. and GELTMAN, S., Phys. Rev. 153 (1967) 4.

7/ HOFSAESS, D., J. Quant. Spectrosc. Radiat. Transfer, 19 (1978) 339 and private communication.

18/ GONGASSIAN, M., Thèse de l'Université de Toulouse, $\mathrm{n}^{\circ} 67$, (1986).

19/ KURUCZ, R.L. and PEYTREMANN, E., "A table of semi-empirical of values" Smithsonian Astrophysical Observatory-Spacial Rep.. 362 (NASA-CR-142879) (1975).

/10/ TRAVING, G., "Plasma Diagnostics" ch II. Lochte-Holtgreven Ed. (1968).

/11/ DRAWIN, H.W. and EMARD, F., Beitr. Plasmaphysik 13 (1973 143.

112/ EVANS , D.L. and TANKIN, A.S. Phys. Fluids 10 (1967) 1137.

/13/ YAKUBOV, I.T., Optics Spectrosc. 19 (1965) 277.

/14/ LIEBERMANN, R.W. and LOWKE, J.J., J. Quant. Spectrosc. Radiat. Transfer, 16 (1976) 253.

/15/ PROULX, P., MOSTAGHIMI, J. and BOULOS, M.I. : 9th Int. Symp. Plasma Chem., Pugnochiuso, Italie, (1989) 89.

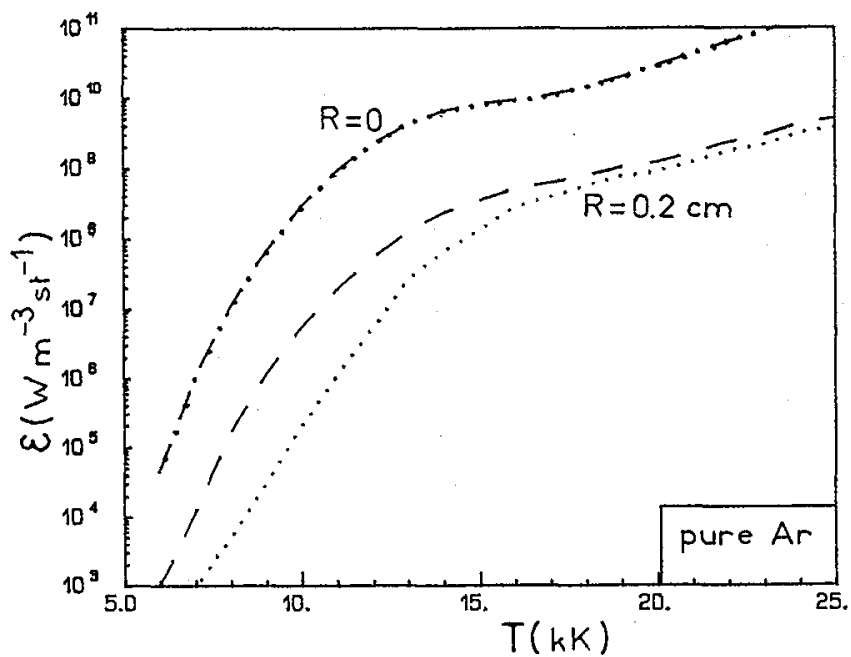

Figure 1 : Emission coefficient for argon ; -- total $; \cdots$ resonance line emission.

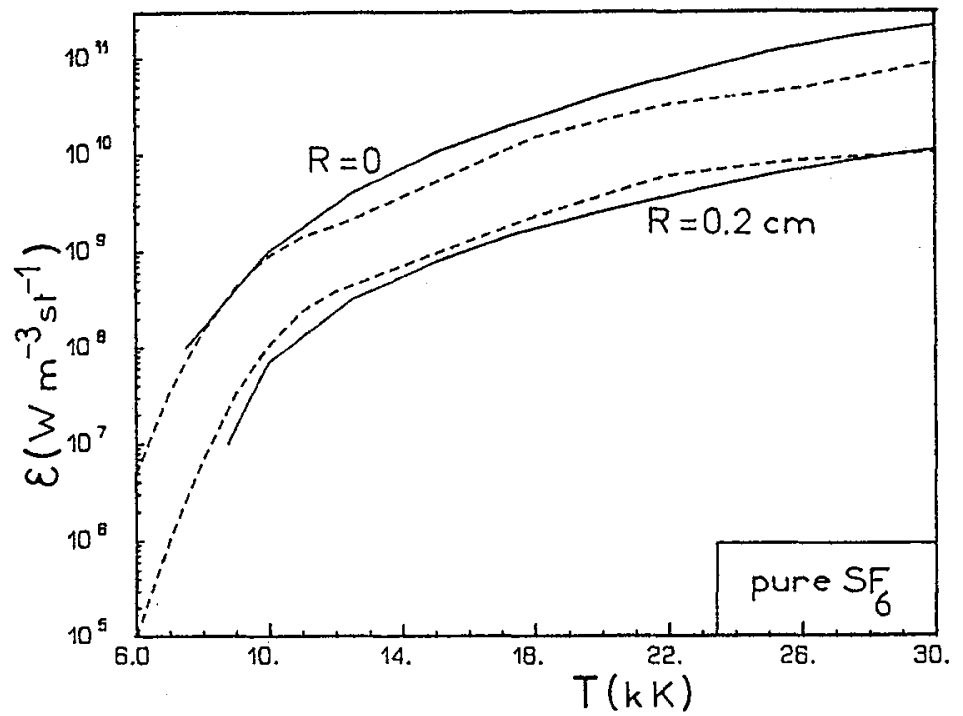

Figure 2 Total emission coefficient for $\mathrm{SF}_{6}: \longrightarrow$ ref. $/ 14 / ;-\cdots$ - . - our results. 


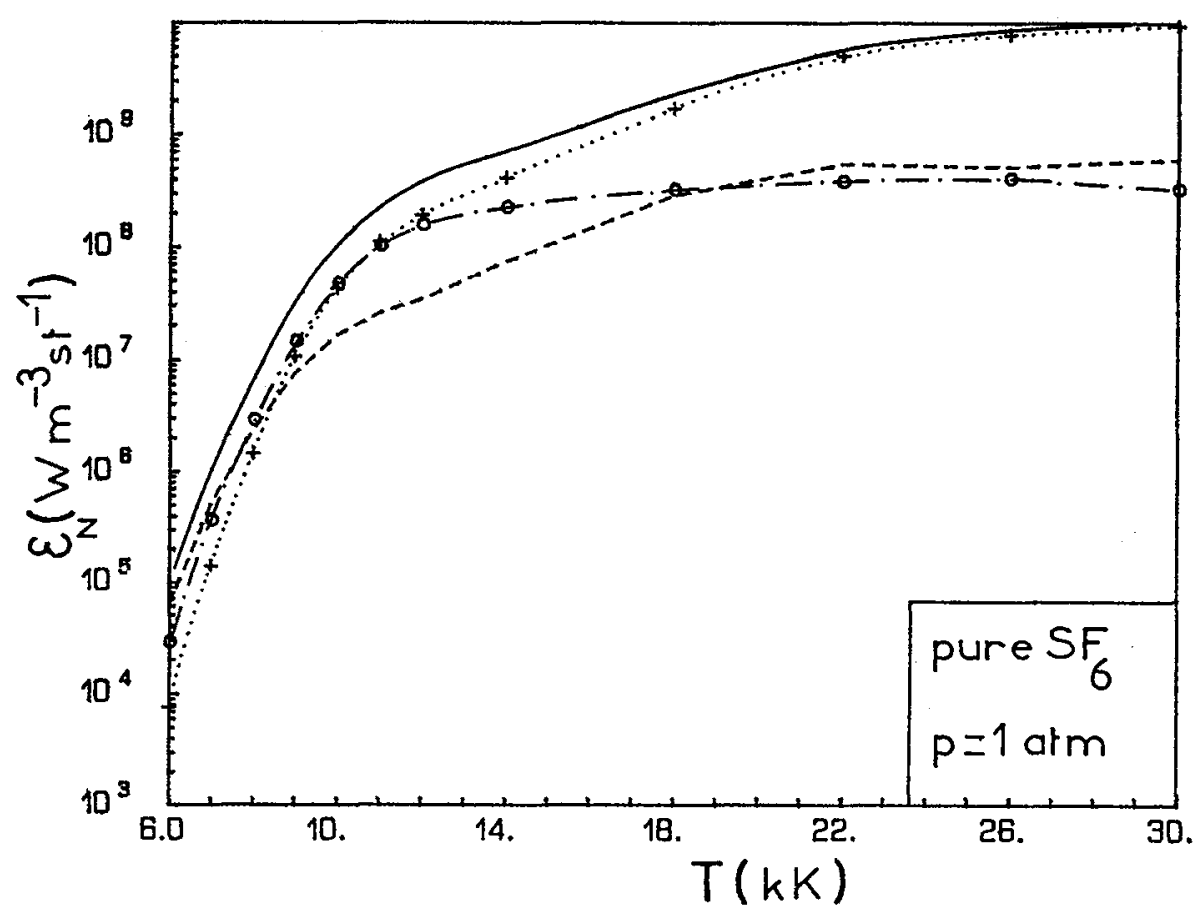

Figure 3 : Components of the net emission coefficient in $\mathrm{SF}_{6}:$ - total emission; $\cdots$ lines $\lambda<200 \mathrm{~nm}$; - - - lines $\lambda>200 \mathrm{~nm}$; - - - continuum.

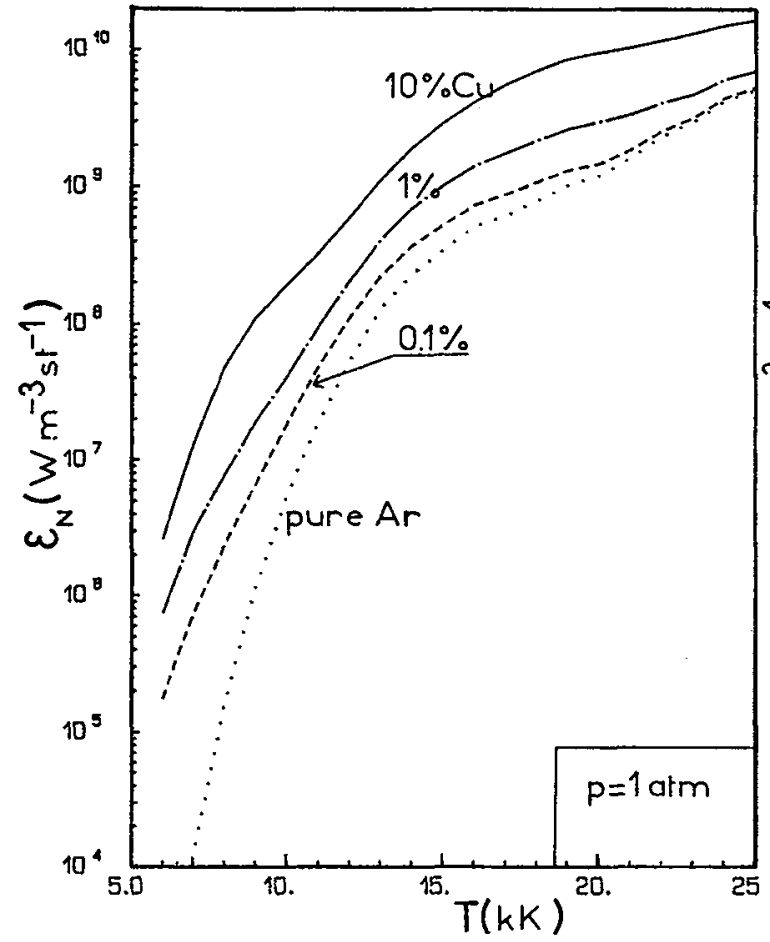

Figure 4 : Influence of copper proportion in the net emission coefficient of Ar-Cu mixture $(\mathrm{R}=0.2 \mathrm{~cm})$.

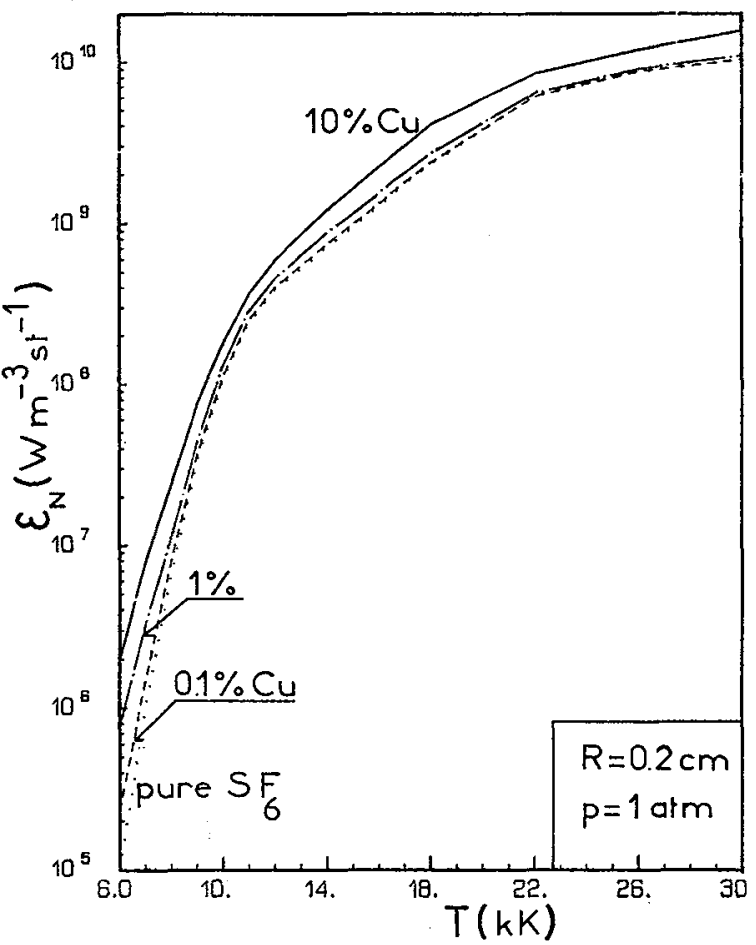

Figure 5: Influence of copper proportion on the net emission coefficient of $\mathrm{SF}_{6}-\mathrm{Cu}$ mixtures. 


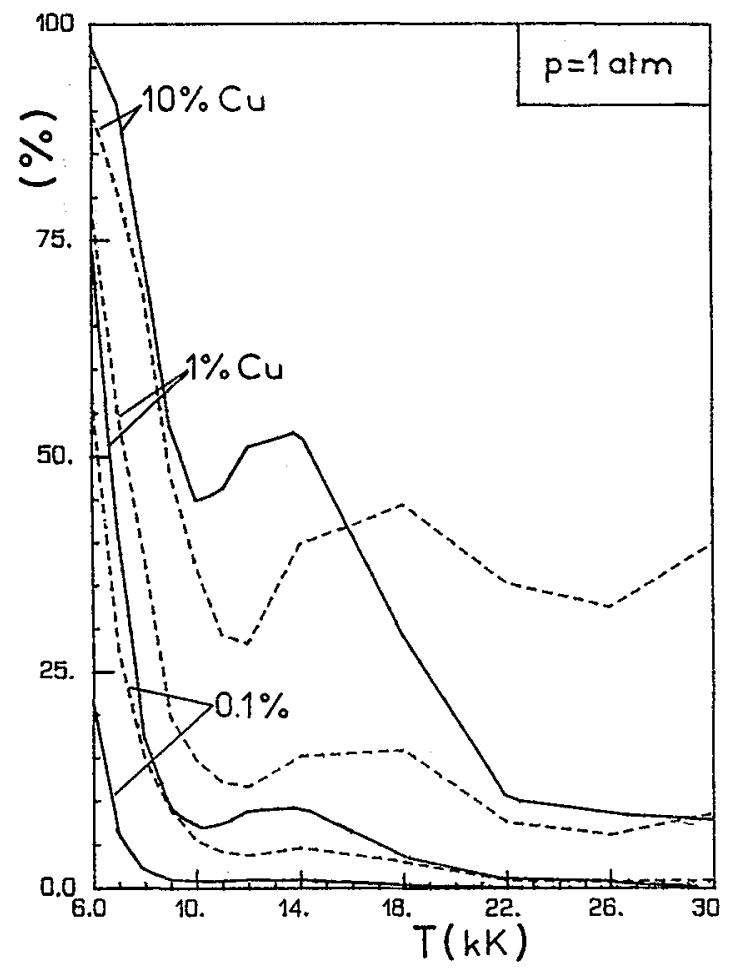

Figure 6 : Relative proportion of copper emission in $\mathrm{SF}_{6}$-Cu mixture : $-\mathrm{R}=0$; ..... $\mathrm{R}=0.5 \mathrm{~cm}$.

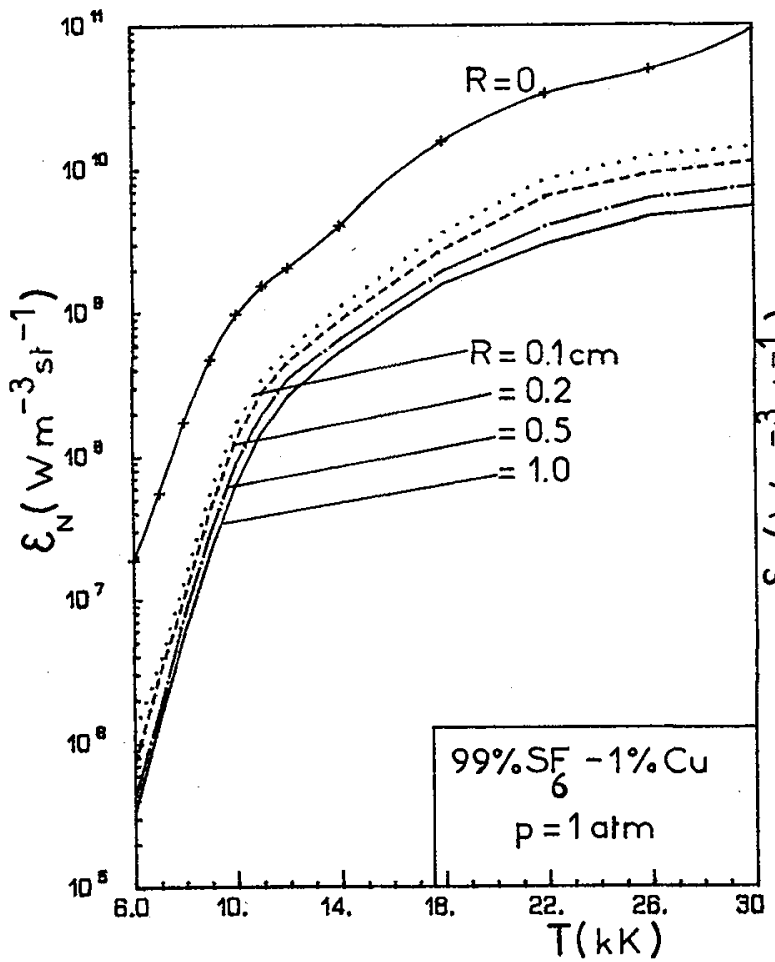

Figure 8: Influence of plasma thickness on the net emission coefficient of $\mathrm{SF}_{6}-\mathrm{Cu}$ mixture.

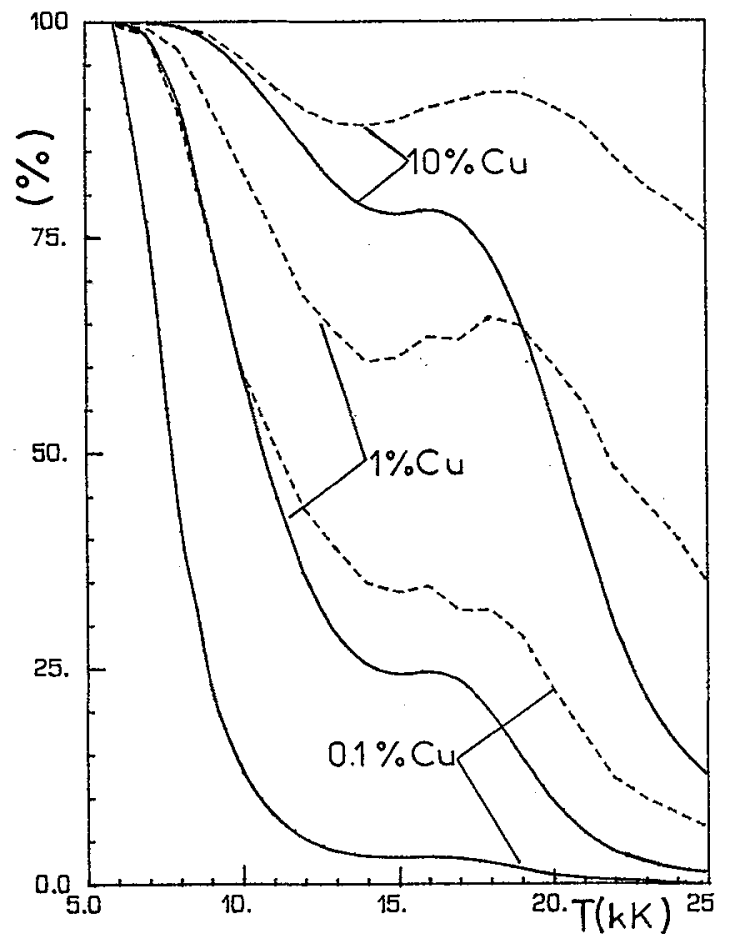

Figure 7 : Relative proportion of copper emission in $\mathrm{Ar}$-Cu mixture : $\mathrm{R}=0$; .... $R=0.5 \mathrm{~cm}$.

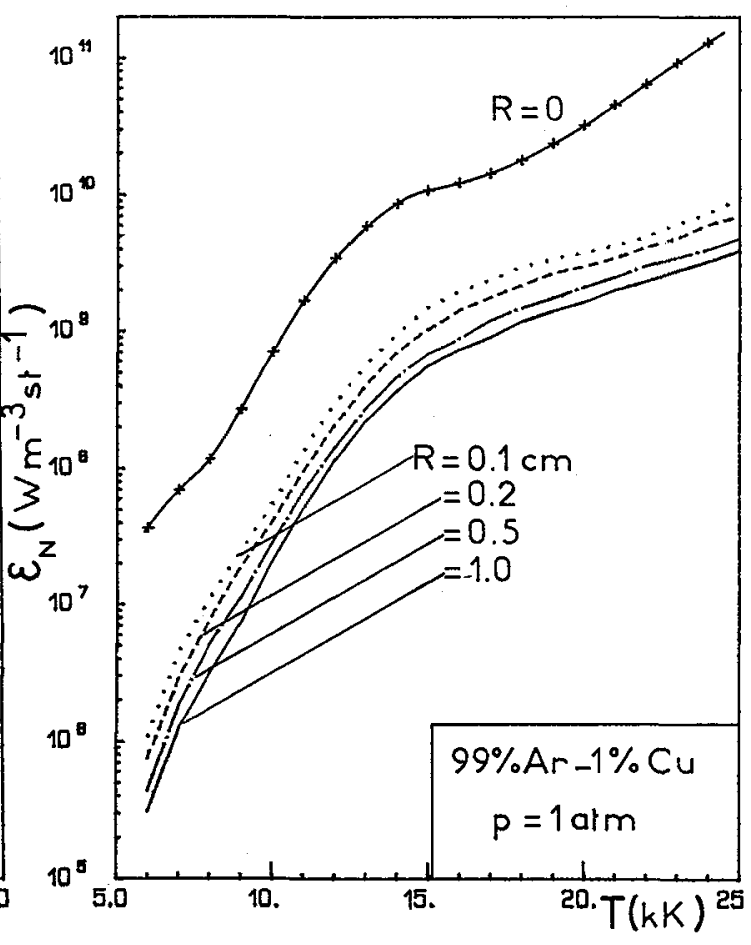

Figure 9: Influence of plasma thickness on the net emission coefficient of Ar-Cu mixture. 


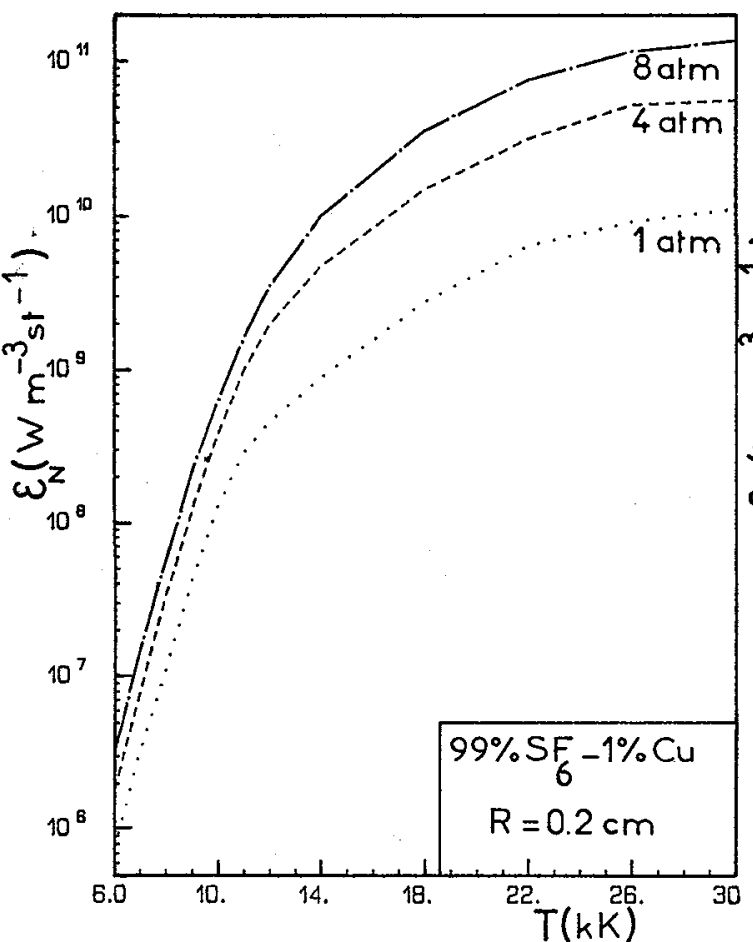

Figure 10: Influence of pressure on the emission of $\mathrm{SF}_{6}$ - Cu mixture.

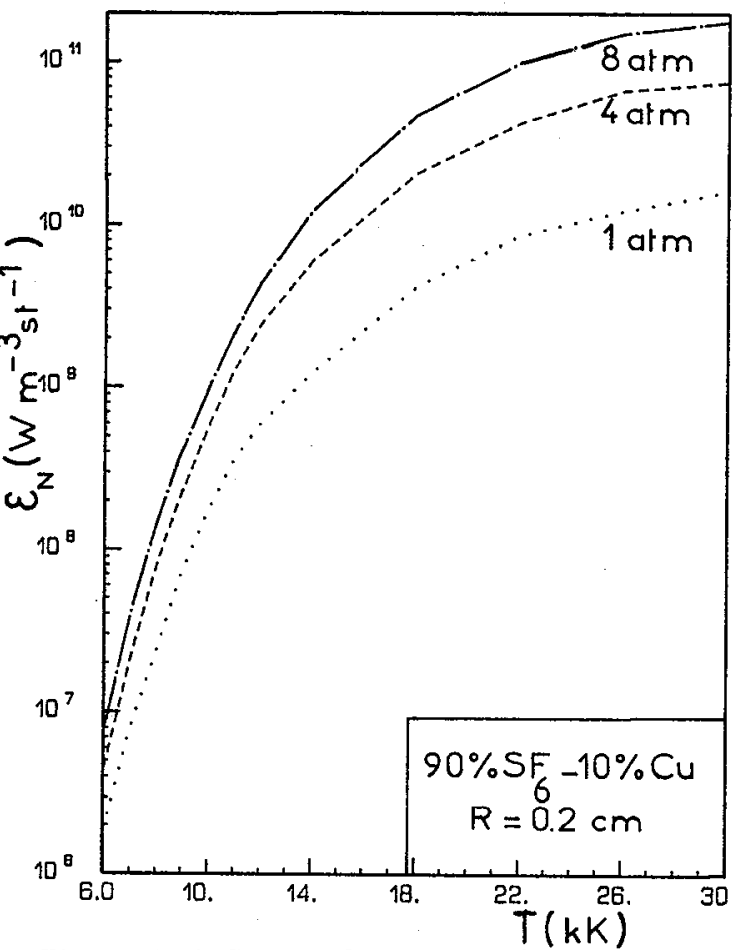

Figure 11: Influence of pressure on the emission of $\mathrm{SF}_{6}$ - $\mathrm{Cu}$ mixture.

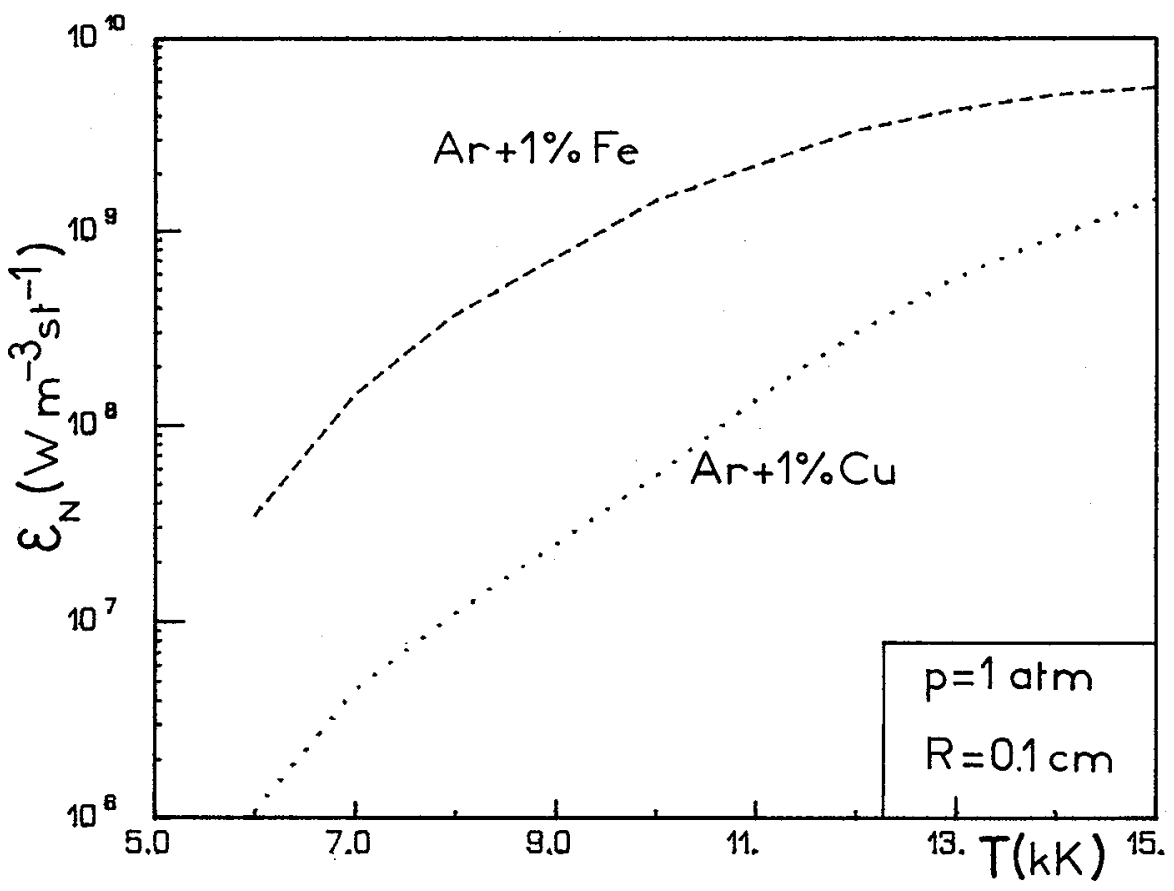

Figure 12 : Comparison between the net emission coefficients of Ar-Cu and $\mathrm{Ar}-\mathrm{Fe}$ (from / 3/. 